\title{
SPIN-POLARIZED PHOTOCURRENT THROUGH QUANTUM DOT PHOTODETECTOR
}

\author{
Nguyen Van Hieu* \\ Institute of Materials Science, VAST \\ College of Technology, Vietnam National University, Hanoi, VNUH
}

Nguyen Bich Ha

College of Technology, Vietnam National University, Hanoi, VNUH

Received 27 November 2006

\begin{abstract}
The theory of the photocurrent through the photodetector based on a two-level semiconductor quantum dot (QD) is presented. The analytical expressions of the matrix elements of the electronic transitions generated by the absorption of the circularly polarized photons are derived in the lowest order of the perturbation theory with respect to the electron tunneling interaction as well as the electron-photon interaction. From these expressions the mechanism of the generation of the spin-polarized of electrons in the photocurrent is evident. It follows that the photodetector based on the two-level semiconductor QD can be used as the model of a source of highly spinpolarized electrons.
\end{abstract}

Keywords: Photocurrent, photodetector, quantum dot, spin-polarized

\section{INTRODUCTION}

With the aim to create the basics of the physics of nanostructured optoelectronic devices containing the semiconductor quantum dots (QDs) as their building blocks the interactions of electrons in QDs with photons and elementary excitations were studied in many experimental and theoretical works. In particular the infrared photodetector based on the intra-conduction band transitions between the $p$-states of the QDs and the wetting layer subband was investigated experimentally [1]. The effects of the charge accumulation on the photocurrent through a QD photodetector were also investigated [2]. In Ref. [3] the infrared photodetectors containing multi-level semiconductor QDs were fabricated and the physical factors influencing the performance of the devices were examined. The effects of the Coulomb blockade on the photocurrent in QD photodetectors were studied in Ref. [4] and the damping of the coherent oscillations in these devices were discussed in Ref. [5]. In the recent theoretical works VillasBôas, Ulloa and Govorov [6], Smirnov and Mourokh [7] have investigated the spin-polarization of the photocurrent generated by the absorption of the polarized light.

In this work we study the theory of the photocurrent through a model photodetector based on the two-level semiconductor QD with the weak tunneling of electrons between the QD and the leads

\footnotetext{
* Corresponding author e-mail: nvhieu@iop.vast.ac.vn
} 
so that the perturbation theory can be applied to the tunneling interaction. For the clarity in the study of the mechanism of the generation of the spin-polarized photocurrent we consider the simple case when the photon energy is not in the resonance with the allowed dipole transition between two discrete levels in the QD and therefore we can apply the perturbation theory not only to the tunneling interaction of electrons, but also to the photon-electron interaction. From the analytical expressions of the matrix elements of the electronic transitions generated by the absorption of the circularly polarized photons it follows the spin-polarization of the photocurrent. It depends on the (conserved) number of electrons in the QD. This result suggests the idea to utilize the photodetector based on the two-level semiconductor QD as a source of highly spin-polarized electrons.

\section{THE MODEL}

Consider a simplest theoretical model of the QD photodetector consisting of a disk-shaped axially symmetric two-level semiconductor QD connected with two semiconducting leads. The QD has two discrete energy levels: the upper level inside the conduction band of the bulk material of the QD with the Bloch wave functions of the form

$$
\left|c_{1 \sigma}\right\rangle=\left|\frac{1}{2}, \pm \frac{1}{2}\right\rangle=\left|S_{\sigma}\right\rangle, \quad \sigma=\uparrow, \downarrow
$$

and the lower one inside its valence band with the Bloch wave functions of the form

$$
\begin{aligned}
& \left|c_{2 \uparrow}\right\rangle=\left|\frac{3}{2}, \frac{3}{2}\right\rangle=\frac{1}{\sqrt{2}}|(X+i Y) \uparrow\rangle, \\
& \left|c_{2 \downarrow}\right\rangle=\left|\frac{3}{2},-\frac{3}{2}\right\rangle=\frac{1}{\sqrt{2}}|(X-i Y) \downarrow\rangle .
\end{aligned}
$$

The leads are the two-dimensional direct band gap semiconductors with the energy band structures to be chosen such that in the presence of some bias voltage applied to two leads the upper level $E_{1}^{c}$ in the QD is lower than the conduction band of the right lead called the lead " 2 " and the lower level $E_{2}^{c}$ in the QD is higher than the valence band of the left lead called the lead "1". Then there exist only the tunnelings between the upper level $E_{1}^{c}$ of the QD and the lead "1" as well as between the lower level $E_{2}^{c}$ of the QD and the lead "2", as it can be seen in Fig.1. The lead " 2 " may be some intrinsic or slightly doped $p$-type semiconductor and works as the source while the lead " 1 " may be some intrinsic or slightly doped $n$-type semiconductor and works as the drain.

Denote $c_{i \sigma}$ or $a_{i \sigma}(\mathbf{k})$ and $c_{i \sigma}^{+}$or $a_{i \sigma}^{+}(\mathbf{k})$ the destruction and creation operators for the electron with the spin projection $\sigma=\uparrow, \downarrow$ at the discrete energy level $E_{i}^{c}$ of the QD or in the lead “ $i$ ", $\mathbf{k}$ being the momentum of electrons in the leads, $\gamma_{\xi}$ and $\gamma_{\xi}^{+}$- those of the photon with the energy $\Omega$ and the polarization characterized by the index $\xi$. The total Hamiltonian of the interacting system of electrons and photons can be written in the form

$$
H=H_{0}+H_{\text {int }}
$$

with

$$
H_{0}=\sum_{i=1}^{2}\left\{E_{i}^{c} \sum_{\sigma} c_{i \sigma}^{+} c_{i \sigma}+U_{i} n_{i \uparrow} n_{i \downarrow}+\sum_{k} E_{i}^{a}(\mathbf{k}) \sum_{\sigma} a_{i \sigma}^{+}(\mathbf{k}) a_{i \sigma}(\mathbf{k})\right\}+V n_{1} n_{2}+\Omega \sum_{\xi} \gamma_{\xi}^{+} \gamma_{\xi},
$$




$$
\begin{aligned}
& \varepsilon_{1}>\varepsilon_{2}, \\
& n_{i \sigma}=c_{i \sigma}^{+} c_{i \sigma}, \quad, \quad \sigma=\uparrow, \downarrow, \\
& n_{i}=n_{i \uparrow}+n_{i \downarrow},
\end{aligned}
$$

where $E_{i}^{a}(\mathbf{k})$ is the energy of the electron with the momentum $\mathbf{k}$ in the lead " $i$ ", $U_{i}$ or $V$ is the potential energy of the Coulomb interaction between two electrons at the same energy level $E_{i}^{c}$ or at different energy levels in the QD, and

$$
\begin{aligned}
& H_{\mathrm{int}}=\sum_{i=1}^{2} \sum_{\sigma}\left\{T_{i}(\mathbf{k}) a_{i \sigma}^{+}(\mathbf{k}) c_{i \sigma}+T_{i}(\mathbf{k})^{*} c_{i \sigma}^{+} a_{i \sigma}(\mathbf{k})\right\} \\
& +\sum_{\sigma, \sigma^{\prime}} \sum_{\xi}\left\{c_{i \sigma}^{+}\left(\lambda_{\xi}\right)_{\sigma, \sigma^{\prime}} c_{2 \sigma^{\prime}} \gamma_{\xi}+\gamma_{\xi}^{+} c_{2 \sigma}^{+}\left(\lambda_{\xi}^{+}\right)_{\sigma \sigma^{\prime}} c_{1 \sigma^{\prime}}\right\},
\end{aligned}
$$

where $T_{i}(\mathbf{k})$ and $T_{i}(\mathbf{k})^{*}$ are the effective tunneling coupling constant, $\left(\lambda_{\xi}\right)_{\sigma \sigma^{\prime}}$ and $\left(\lambda_{\xi}^{+}\right)_{\sigma \sigma^{\prime}}$ are the matrix elements of the allowed dipole electromagnetic transitions of the electrons between two energy levels
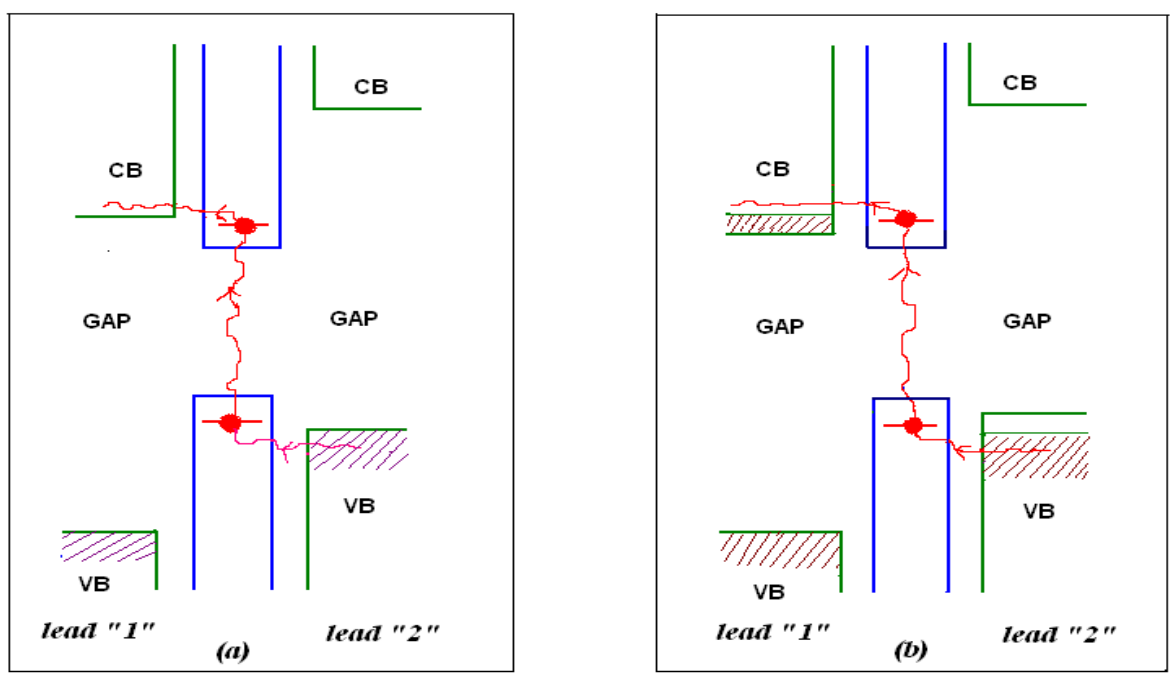

Fig. 1: Schematic energy band structure of the $Q D-$ Photodetector. Dashed areas: the bands of occupied energy levels. Wave lines: virtual transitions of the electrons from the occupied levels in the valence band of the right lead "2" to the lower discrete energy level in the $Q D$ due to the tunneling; from the lower discrete energy level in the $Q D$ to the upper one due to the interaction with the electromagnetic field and from the upper discrete energy level in the $Q D$ to the empty levels in the conduction band of the left lead " 1 " due to the tunneling. (a) The leads are made from intrinsic direct band gap semiconductors. (b) The lead "1" is made from a slightly doped n-type semiconductor while the lead " 2 " is made from a slightly doped p-type semiconductor

\section{MATRIX ELEMENTS OF THE PHOTON ABSORPTION INDUCED TRANSITION PROCESSES}

The total electron number $n$ in the QD may have five different values $n=0,1,2,3$ and 4 . In the 
transition process of an electron from the lead " 2 " through the QD to the lead "1" generated by the absorption of a photon this number $n$ remains unchanged. In each special case with $n=0$ or $n=4$ there exists only one state: the vacuum $|0\rangle$ for $n=0$ and the 4-electron state $\left|c_{1 \uparrow} c_{1 \downarrow} c_{2 \uparrow} c_{2 \downarrow}\right\rangle$ for $n=4$. In each case with $n=1$ or $n=3$ there are two degenerate ground states of the electron system in the QD: $\left|c_{2 \uparrow}\right\rangle$ and $\left|c_{2 \downarrow}\right\rangle$ for $n=1$ and $\left|c_{1 \uparrow} c_{2 \uparrow} c_{2 \downarrow}\right\rangle$ and $\left|c_{1 \downarrow} c_{2 \uparrow} c_{2 \downarrow}\right\rangle$ for $n=3$. In the case with $n=2$ there is only one ground state $\left|c_{2 \uparrow} c_{2 \downarrow}\right\rangle$. Denote $\left|\varphi_{i}\right\rangle$ and $\left|\varphi_{f}\right\rangle$ the state vectors of the electron system of the QD in the initial and final states of each transition process. In the lowest (third) order of the perturbation theory with respect to the interaction Hamiltonian (3) the matrix element of the transition process of the electron with the spin projection $\sigma_{2}$ and the momentum $\mathbf{k}_{2}$ in the lead "2" through the QD into the electron with the spin projection $\sigma_{1}$ and the momentum $\mathbf{k}_{1}$ in the lead "1" due to the absorption of the photon with the energy $\Omega$ and the polarization index $\xi$ can be presented in the form

$$
\begin{aligned}
& \left\langle\varphi_{f}, a_{1 \sigma_{1}}\left(\mathbf{k}_{\mathbf{1}}\right)|S| a_{2 \sigma_{2}}\left(\mathbf{k}_{2}\right), \gamma_{\xi}, \varphi_{i}\right\rangle= \\
& -i 2 \pi \delta\left[E_{1}^{a}\left(\mathbf{k}_{\mathbf{1}}\right)-E_{2}^{a}\left(\mathbf{k}_{2}\right)-\Omega\right] T_{1}\left(\mathbf{k}_{\mathbf{1}}\right) T_{2}\left(\mathbf{k}_{2}\right)^{*}\left\langle\varphi_{f}\left|R\left(\mathbf{k}_{\mathbf{1}}, \sigma_{1} ; \mathbf{k}_{2}, \sigma_{2} ; \Omega, \xi\right)\right| \varphi_{i}\right\rangle .
\end{aligned}
$$

It is straightforward to derive the expressions of $\left\langle\varphi_{f}\left|R\left(\mathbf{k}_{\mathbf{1}}, \sigma_{1} ; \mathbf{k}_{2}, \sigma_{2} ; \Omega, \xi\right)\right| \varphi_{i}\right\rangle$ in each case $n=0,1,2,3$ and 4 .

Case $1, n=0$.

$$
\left\langle 0\left|R\left(\mathbf{k}_{\mathbf{1}}, \sigma_{1} ; \mathbf{k}_{\mathbf{2}}, \sigma_{2} ; \Omega, \xi\right)\right| 0\right\rangle=-\frac{\left(\lambda_{\xi}\right)_{\sigma_{1} \sigma_{2}}}{E_{1}^{c}-E_{2}^{c}-\Omega}\left[\frac{1}{E_{1}^{c}+i \Gamma-E_{1}^{a}\left(\mathbf{k}_{\mathbf{1}}\right)}-\frac{1}{E_{2}^{c}+i \Gamma-E_{2}^{a}\left(\mathbf{k}_{\mathbf{2}}\right)}\right]
$$

Case 2, $n=1$.

$$
\begin{aligned}
& \left\langle c_{2 \sigma_{f}}\left|R\left(\mathbf{k}_{\mathbf{1}}, \sigma_{1} ; \mathbf{k}_{\mathbf{2}}, \sigma_{2} ; \Omega, \xi\right)\right| c_{2 \sigma_{i}}\right\rangle= \\
& -\left[\frac{\delta_{\sigma_{i} \sigma_{f}} \delta_{-\sigma_{i} \sigma_{f}}\left(\lambda_{\xi}\right)_{\sigma_{1} \sigma_{2}}-\delta_{-\sigma_{i} \sigma_{f}} \delta_{-\sigma_{i} \sigma_{2}}\left(\lambda_{\xi}\right)_{\sigma_{1}-\sigma_{2}}}{E_{1}^{c}+V-E_{2}^{c}-U_{2}-\Omega}+\frac{\delta_{\sigma_{f} \sigma_{2}}\left(\lambda_{\xi}\right)_{\sigma_{1} \sigma_{i}}}{E_{1}^{c}-E_{2}^{c}-\Omega}\right] \\
& \times\left[\frac{1}{E_{1}^{c}+i \Gamma+V-E_{1}^{a}\left(\mathbf{k}_{\mathbf{1}}\right)}-\frac{1}{E_{2}^{c}+i \Gamma+U_{2}-E_{2}^{a}\left(\mathbf{k}_{\mathbf{2}}\right)}\right] .
\end{aligned}
$$

Case 3, $n=2$.

$$
\begin{aligned}
& \left\langle c_{2 \downarrow} c_{2 \uparrow}\left|R\left(\mathbf{k}_{\mathbf{1}}, \sigma_{1} ; \mathbf{k}_{\mathbf{2}}, \sigma_{2} ; \Omega, \xi\right)\right| c_{2 \uparrow} c_{2 \downarrow}\right\rangle= \\
& \frac{\left(\lambda_{\xi}\right)_{\sigma_{1} \sigma_{2}}}{E_{1}^{c}+V-E_{2}^{c}-U_{2}-\Omega}\left[\frac{1}{E_{1}^{c}+i \Gamma+V-E_{1}^{a}\left(\mathbf{k}_{\mathbf{1}}\right)}-\frac{1}{E_{2}^{c}+i \Gamma+U_{2}-E_{2}^{a}\left(\mathbf{k}_{\mathbf{2}}\right)}\right] .
\end{aligned}
$$

Case 4, $n=3$. 


$$
\begin{aligned}
& \left\langle c_{1 \sigma_{f}} c_{2 \downarrow} c_{2 \uparrow}\left|R\left(\mathbf{k}_{\mathbf{1}}, \sigma_{1} ; \mathbf{k}_{2}, \sigma_{2} ; \Omega, \xi\right)\right| c_{2 \uparrow} c_{2 \downarrow} c_{1 \sigma_{i}}\right\rangle= \\
& -\left[\frac{\delta_{\sigma_{i} \sigma_{1}}\left(\lambda_{\xi}\right)_{\sigma_{f} \sigma_{2}}}{E_{1}^{c}+V-E_{2}^{c}-U_{2}-\Omega}+\frac{\delta_{\sigma_{f} \sigma_{i}} \delta_{-\sigma_{f} \sigma_{i}}\left(\lambda_{\xi}\right)_{\sigma_{1} \sigma_{2}}-\delta_{-\sigma_{f} \sigma_{i}} \delta_{-\sigma_{f} \sigma_{1}}\left(\lambda_{\xi}\right)_{-\sigma_{1} \sigma_{2}}}{E_{1}^{c}+U_{1}-E_{2}^{c}-U_{2}-\Omega}\right] \\
& \times\left[\frac{1}{E_{1}^{c}+i \Gamma+U_{1}+2 V-E_{1}^{a}\left(\mathbf{k}_{1}\right)}-\frac{1}{E_{2}^{c}+i \Gamma+U_{2}+V-E_{2}^{a}\left(\mathbf{k}_{2}\right)}\right] .
\end{aligned}
$$

Case $5, n=4$.

$$
\begin{aligned}
& \left\langle c_{1 \downarrow} c_{1 \uparrow} c_{2 \downarrow} c_{2 \uparrow}\left|R\left(\mathbf{k}_{\mathbf{1}}, \sigma_{1} ; \mathbf{k}_{\mathbf{2}}, \sigma_{2} ; \Omega, \xi\right)\right| c_{2 \uparrow} c_{2 \downarrow} c_{1 \uparrow} c_{1 \downarrow}\right\rangle= \\
& -\frac{\left(\lambda_{\xi}\right)_{\sigma_{1} \sigma_{2}}}{E_{1}^{c}+U_{1}-E_{2}^{c}-U_{2}-\Omega}\left[\frac{1}{E_{1}^{c}+i \Gamma+U_{1}-E_{1}^{c}\left(\mathbf{k}_{\mathbf{1}}\right)}-\frac{1}{E_{2}^{c}+i \Gamma+U_{2}-E_{2}^{c}\left(\mathbf{k}_{2}\right)}\right] .
\end{aligned}
$$

In the formulae (5) - (9) the broadening of the discrete energy levels in the QD due to the electron tunneling between the leads and the QD was taken into accound by introducing the half widths of the discrete energy levels which have approximately the same value $\Gamma$.

\section{SPIN POLARIZATION OF THE PHOTOCURRENT}

Suppose that the QD photodetector is irradiated by the circularly polarized light propagating along the symmetry axis $\mathrm{O} z$ of the QD and having the photon spin projection $s_{z}=-1$ on the direction of this axis. Denote $\lambda$ the corresponding matrix $\lambda_{\xi}$ in the interaction Hamiltonian. The absorption of a photon with the spin projection $s_{z}=-1$ on the $\mathrm{O} z$ axis induces the transition of an electron from the state $\left|c_{2 \uparrow}\right\rangle=\left|\frac{3}{2}, \frac{3}{2}\right\rangle$ on the lower energy level to the state $\left|c_{1 \uparrow}\right\rangle=\left|\frac{1}{2}, \frac{1}{2}\right\rangle$ on the upper energy level of the QD. The matrix $\lambda$ has only one non-vanishing matrix element:

$$
\begin{aligned}
& \lambda_{\uparrow \uparrow}=g \neq 0, \\
& \left(\lambda_{\uparrow \downarrow}\right)=\left(\lambda_{\downarrow \uparrow}\right)=\left(\lambda_{\downarrow \downarrow}\right)=0 .
\end{aligned}
$$

From the expressions (5), (6), (7) and (9) of the transition amplitudes in four cases with $n=0$, $n=1, n=2$ and $n=4$ it follows that they do not vanish only if $\sigma_{1}=\uparrow:$ the outgoing electrons are totally polarized and have the spin projection $\sigma_{1}=\uparrow$.

Consider the case $n=3$ and for clarity introduce the brief notation

$$
\left|\left\langle c_{1 \sigma_{f}} c_{2 \downarrow} c_{2 \uparrow}\left|R\left(\mathbf{k}_{\mathbf{1}}, \sigma_{1} ; \mathbf{k}_{\mathbf{2}}, \sigma_{2} ; \Omega, \xi\right)\right| c_{2 \uparrow} c_{2 \downarrow} c_{1 \sigma_{i}}\right\rangle\right|^{2}=W_{\sigma_{f}}^{\sigma_{1} \sigma_{i}} .
$$

From the expression (8) it follows that there exists only three non-vanishing squared amplitude moduli $W_{\downarrow \downarrow}^{\uparrow \uparrow}, W_{\uparrow \uparrow}^{\uparrow \uparrow}$ and $W_{\uparrow \downarrow}^{\downarrow \uparrow}$ of three processes with different configurations of the spin projections of the electrons in the QD and the incoming and outgoing electrons. In the transition processes described by the squared amplitude moduli $W_{\downarrow \downarrow}^{\uparrow \uparrow}$ and $W_{\uparrow \uparrow}^{\uparrow \uparrow}$ the spin projection of the 
electron at the upper energy level $E_{1}^{c}$ of the QD remains unchanged, $\sigma_{i}=\sigma_{f}$, while in the transition process described by the squared amplitude modulus $W_{\uparrow \downarrow}^{\downarrow \uparrow}$ the spin projection of this electron must be changed from $\sigma_{i}=\downarrow$ to $\sigma_{f}=\uparrow$. From the rate equations it follows that after the establishment of the steady state the electron at the upper energy level $E_{1}^{c}$ of the QD must have the constant spin projection $\sigma_{i}=\sigma_{f}=\uparrow$. Then only the transition process with the squared amplitude modulus $W_{\uparrow \uparrow}^{\uparrow \uparrow}$ takes place and the outgoing electrons also have only one spin projection $\sigma_{1}=\uparrow$.

Thus we have shown that the photocurrent induced by the absorption of the photons with the spin projection $s_{z}=-1$ is totally polarized, the spin polarization of the outgoing electrons being $\sigma_{1}=\uparrow$, and the QD photodetector may be used as a source of spin polarized electrons.

\section{CONCLUSION AND DISCUSSIONS}

We have shown that in the lowest order of the perturbation theory with respect to both the photon-electron interaction and the effective lead-dot tunneling interaction the photocurrent through the proposed model quantum dot photodetector induced by the absorption of the photons with the spin projection $s_{z}=-1$ is totally spin polarized, the spin polarization of the outgoing electrons being $\sigma_{1}=\uparrow$, and the QD photodetector may be used as a source of spin polarized electrons. However, the formulae (5) - (9) of the transition amplitudes are valid if and only if the expressions of the denominators in the r.h.s of these formulae do not vanish, that is the radiation frequency $\Omega$ is out of the resonance with the dipole electromagnetic transition between two corresponding states of the electron system in the QD:

$$
\begin{aligned}
& \Omega \neq E_{1}^{c}-E_{2}^{c}, \\
& \Omega \neq E_{1}^{c}+V-E_{2}^{c}-U_{2}, \\
& \Omega \neq E_{1}^{c}+U_{1}-E_{2}^{c}-U_{2}
\end{aligned}
$$

If some condition among the conditions (11) is not satisfied, then we must take into account the contributions of the corresponding high order terms of the perturbation theory. Moreover, the real spin structure of the wave function of the electron in the lower energy level is more complicated than that in the proposed model: there is the band mixing. The effects of the high order terms of the perturbation series and the band mixing would be the subjects of the further studies.

\section{ACKNOWLEDGEMENT}

The authors would like to express their deep gratitude to the Ministry of Science and Technology for the support of the National Basic Research Program in Natural Sciences.

\section{REFERENCES}

1. Chu, L., Zrenner, A., Bougeard, D., Bichler, M., and Abstreiter, G. (2002), Physica E 13, 301.

2. Monte, A.F.G., Finley, J.J., Itskevitch, I., Skolnick, M.S., Mowbray, D.J., and Hopkins, M. (2003), Physica E 17, 37. 
3. Lim, H., Zhang, W., Tsao, S., Sills, T., Szafraniec, J., Mi, K., Movaghar, B., and Razeghi, M. (2005) Phys. Rev. B 72, 085332.

4. Kuo, D.M.-T. and Chang, Y.-C. (2003), Phys. Rev. B 67, 035313.

5. Villas-Bôas, J.M., Ulloa, S.E., and Govorov, A.O. (2005), Physica E 26, 337.

6. Villas-Bôas, J.M., Ulloa, S.E., and Govorov, A.O. (2005), Cond-Mat. 1, 050973.

7. Smirnov, A.Y. and Mourokh, L.G. (2005), Phys. Rev. B 71, 161305. 